\title{
Resolution limits of migration and linearized waveform inversion images in a lossy medium
}

\author{
Gerard T. Schuster, Gaurav Dutta and Jing Li \\ Department of Earth Science and Engineering, King Abdullah University of Science and Technology (KAUST), Thuwal 23955-6900, Kingdom of Saudi \\ Arabia.E-mail: inter.lijing@gmail.com
}

Accepted 2017 March 10. Received 2017 March 9; in original form 2016 June 20

\begin{abstract}
SUMMAR Y
The vertical- and horizontal-resolution limits $\Delta x^{\text {lossy }}$ and $\Delta z^{\text {lossy }}$ of post-stack migration and linearized waveform inversion images are derived for lossy data in the far-field approximation. Unlike the horizontal resolution limit $\Delta x \propto \lambda z / L$ in a lossless medium which linearly worsens in depth $z, \Delta x^{\text {lossy }} \propto z^{2} / Q L$ worsens quadratically with depth for a medium with small $Q$ values. Here, $Q$ is the quality factor, $\lambda$ is the effective wavelength, $L$ is the recording aperture, and loss in the resolution formulae is accounted for by replacing $\lambda$ with $z / Q$. In contrast, the lossy vertical-resolution limit $\Delta z^{\text {lossy }}$ only worsens linearly in depth compared to $\Delta z \propto \lambda$ for a lossless medium. For both the causal and acausal $Q$ models, the resolution limits are linearly proportional to $1 / Q$ for small $Q$. These theoretical predictions are validated with migration images computed from lossy data.
\end{abstract}

Key words: Controlled source seismology; Seismic attenuation; Theoretical seismology; Wave scattering and diffraction.

\section{INTRODUCTION}

All seismic imaging methods should include formulae for the theoretical bounds of spatial resolution in the inverted image. In this way, experimentalists can design the optimal geophysical survey that achieves the desired resolution. These limits also dictate the smallest cell size in the final tomogram or migration image.

The theory of spatial resolution has been established for migration, inversion (Beylkin et al. 1985; Cohen et al. 1986; Bleistein 1987), diffraction tomography (Wu \& Toksoz 1987), traveltime tomography (Williamson \& Worthington 1993; Sheng \& Schuster 2003; Dahlen 2004) and linearized waveform tomography (Mora 1989; Huang \& Schuster 2014). For example, formulae for lateral resolution of migrated seismic images are presented by Berkhout \& Van Wulfften (1979), Berkhout (1984), Safar (1985) and Chen \& Schuster (1999). Such resolution formulae are especially important when the exploration prospects are stratigraphic and not structural. For stratigraphic traps, the subtle changes in lithology, for example, sandstone-to-shale variations, are indicated by small changes in the propagation velocity within a quasi-horizontal layer. Thus the lateral resolution in the stratigraphic image is crucial for determining the uncertainty in the estimate of economic reserves.

Many papers have presented resolution formulae for 2-D seismic surveys, some realistic and some achieved only in a laboratory setup. Beylkin et al. (1985) study the resolution of 2-D surveys using the Born approximation. They show the connection between the maximum wavenumber in the data and the spatial resolution of the migrated image. Their formulae reveal how the resolution limit is a function of the parameters of the seismic survey. Carrion et al. (1991) present a simplified and practical theory for judging the image resolution capability of limited aperture migration in three dimensions. However, their examples were all within a 2-D framework with conventional seismic acquisition surveys. Wu \& Toksoz (1987) show the relationship between the source-receiver geometry and image resolution. Witten \& Molyneux (1988) use vertical seismic profile-type recording layouts and diffraction tomography to study the resolution limits for imaging scatterers with small contrasts in impedance. Mora (1989) describes the tomographic term associated with waveform inversion and discusses its resolution properties, and Williamson \& Worthington (1993) derive formulae for frequency-limited resolution in ray-based traveltime tomography. The extension of these limits to waveform traveltime tomography is described by Sheng \& Schuster (2003) and Dahlen (2004). An overview by Huang \& Schuster (2014) summarizes the resolution limits dictated by the tomographic and migration gradients associated with migration and linearized waveform inversion.

These previous studies have only considered resolution limits in lossless sediments, and do not consider the effects of attenuation in lossy media. Such effects can be very important in degrading image resolution when reflections propagate through unconsolidated soil and semi-consolidated sediments filled with gas. To rectify this deficiency, we now derive the far-field resolution limits for post-stack migrated images in lossy media. We show that they are applicable to linearized inversion images and define the lower bounds of resolution for pre-stack migration images.

This paper is organized into four sections. Following the introduction we present the theory for the resolution limits of a point source 
(a) Acausal Mag. Spectrum

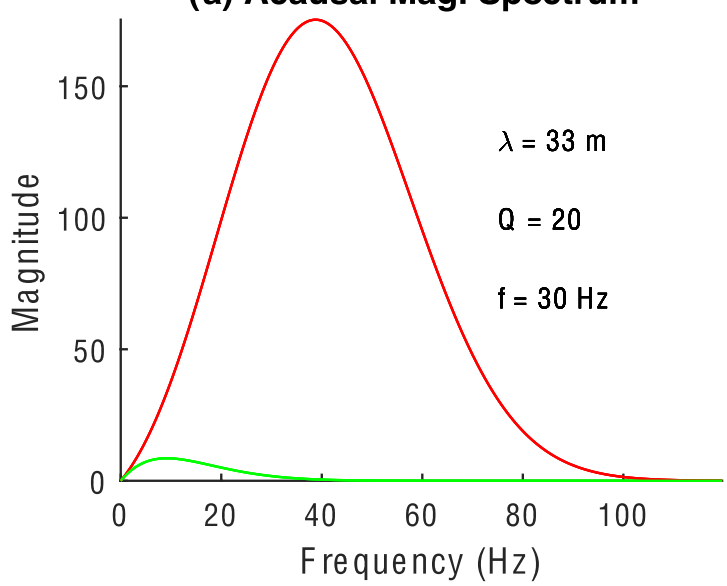

(c) Causal Mag. Spectrum

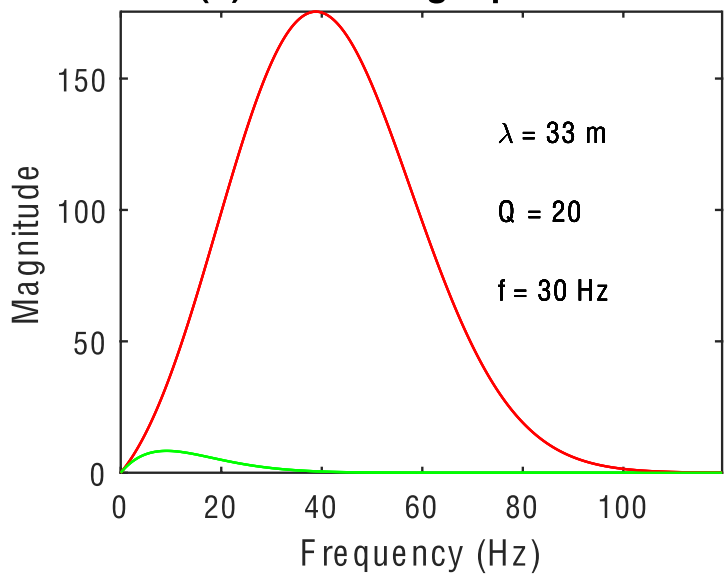

(b) Acausal Seismograms

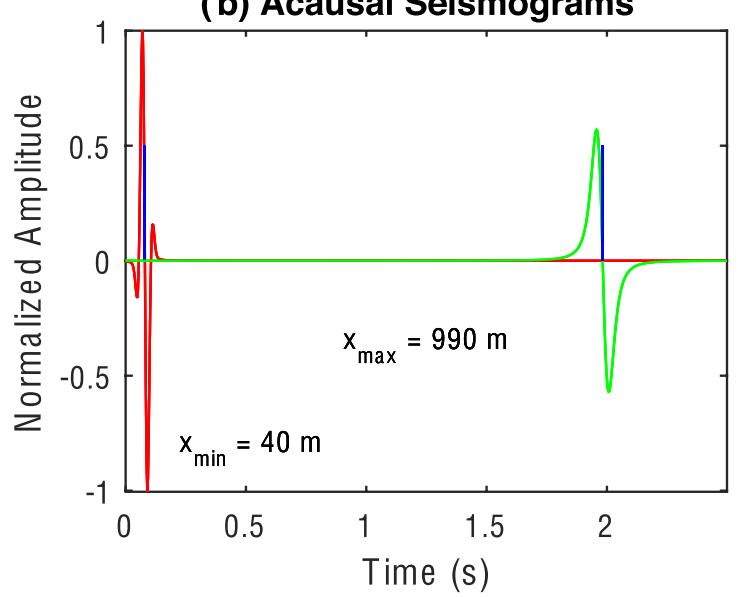

(d) Causal Seismograms

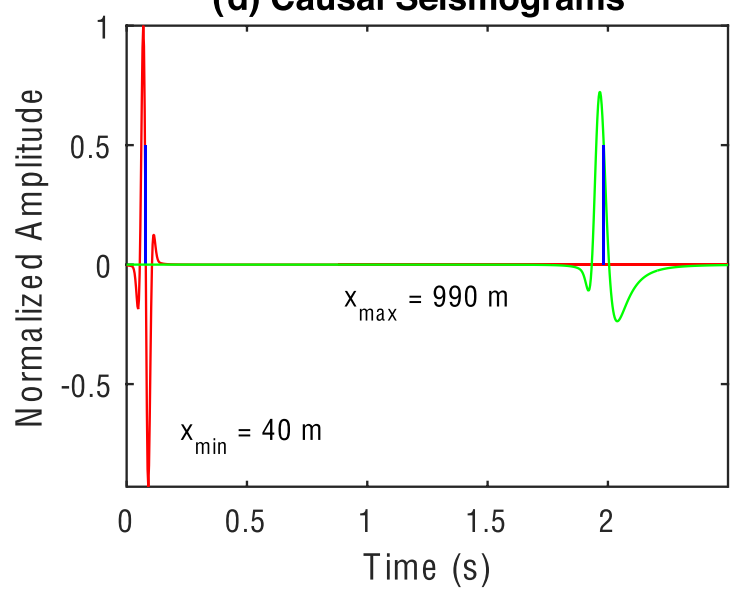

Figure 1. (a) Spectra for near-offset (red curve with $40 \mathrm{~m}$ source-receiver offset) and far-offset (green curve with $990 \mathrm{~m}$ source-receiver offset) traces and (b) the corresponding seismograms for the constant $Q=20$ and constant velocity $\left(c=1 \mathrm{~km} \mathrm{~s}^{-1}\right)$ model. (c) and (d) are the same as the top row of figures except they are for the causal Q model where $Q=20$ but the velocity depends on frequency so that, unlike the acausal arrivals in (b), the lower frequencies arrive after the higher frequencies. The amplitudes of the green traces have been increased by a factor of 30 , and the vertical blue bar indicates the time of arrival of an impulse travelling at $1 \mathrm{~km} \mathrm{~s}^{-1}$.

and a point scatterer in a homogeneous lossy medium. Here, the input data are the post-stack traces, and the output is the post-stack migration image. Resolution formulae are derived for both causal and acausal Q models. The causal Q model constrains waves to propagate no faster than the actual speed of propagation, while the acausal Q model violates this constraint. As an example, Fig. 1(b) presents acausal seismograms compared to the causal ones in Fig. 1(d). We also discuss how the migration formulae for the Q model can be extended to images computed from linearized inversion. The predicted limits of resolution are validated in the next section by migrating post-stack data for a buried point source. In particular, our numerical simulations validate the theoretical predictions that $\Delta_{x}^{\text {lossy }}$ worsens as $z_{o}^{2} / Q$ and $\Delta_{z}^{\text {lossy }}$ worsens as $z_{o} / Q$ where $z_{o}$ is the depth of the scatterer for post-stack data. The last section presents conclusions and a discussion.

\section{THEORY}

The goal is to derive the lossy resolution limits of a post-stack migration image by using the migration stretch procedure described in Chen \& Schuster (1999). These post-stack limits also define the lower bounds of resolution for pre-stack migration images.

\subsection{Migration stretch limits for lossless media}

The diffraction stack formula (French 1974) for migrating 2-D poststack data is given as

$m(\mathbf{x})=\int_{-L}^{L} d\left(\mathbf{g}, \tau_{g x g}\right) \mathrm{d} x_{g}$

where $m(\mathbf{x})$ represents the post-stack migration image at the trial image point $\mathbf{x}$, the geophones are located at $\mathbf{g}=\left(x_{g}, 0\right), d(\mathbf{g}, t)$ is the zero-offset ( $\mathrm{ZO}$ ) trace and $\tau_{\text {gxg }}$ is the two-way zero-offset traveltime for waves to propagate from the source position at $\mathbf{g}$ down to the trial image point at $\mathbf{x}$, and back up to the geophone position at $\mathbf{g}$ co-located with the source. Here, the normalized poststack seismic data are recorded along the horizontal recording line of length $2 L$, geometrical spreading losses are corrected in the data, $c$ is the subsurface homogeneous velocity, and the spatial sampling of traces is continuous. The trace $d(\mathbf{g}, t)$ has an implicit doubletime differentiation and muting of the direct arrivals. It is assumed that corrections have been applied to the post-stack data so that the records are equivalent to traces recorded by an experiment where there is zero-offset between the source and receiver. 
Poststack Migration: Smear and Sum Refl. Amp. Along Circles

(a) One-trace migration

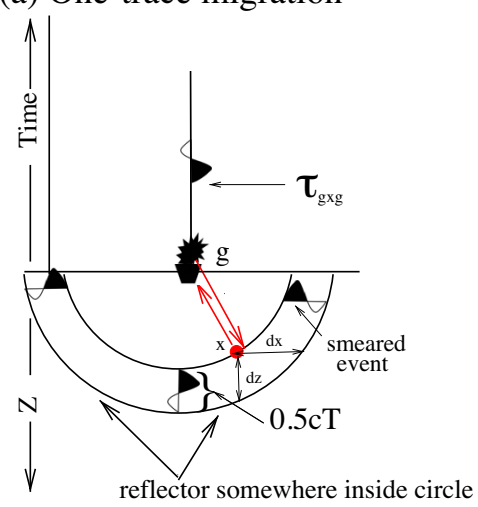

(b) Two-trace migration

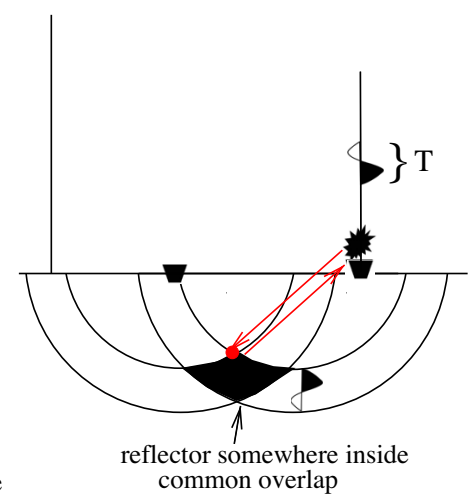

Figure 2. The migration image for each trace is computed by smearing and summing the trace amplitudes along the appropriate fat doughnuts in $(x, z)$ (Claerbout 1992). Migration of two traces in (b) has better spatial resolution than migrating just one trace in (a), and the minimum thickness of each fat doughnut is $0.5 c T$, where $T$ is the dominant period of the source wavelet and $c$ is the propagation velocity.

For a single source and single receiver the above equation becomes

$m(\mathbf{x})=d\left(\mathbf{g}, \tau_{g x g}\right)$,

where the wavelet in $d\left(\mathbf{g}, \tau_{\text {gxg }}\right)$ with dominant period $T$ gets mapped into the migration doughnut in Fig. 2(a). We conservatively define the radial-resolution limit of the migration image to be proportional to the radial thickness $\lambda / 2$ of this doughnut, where $\lambda=c T$. The horizontal width $d x$ of the doughnut at the image point $\mathbf{x}$ defines the horizontal-resolution limit of the migration operator at $\mathbf{x}$. Therefore, the smallest value of $d x$ will be associated with the trace that is farthest offset from $\mathbf{x}$ because the corresponding doughnut has the minimum-horizontal cut at $\mathbf{x}$. This minimum value of $d x$ at $\mathbf{x}$ is defined to be the horizontal-resolution limit $\Delta x$ (Chen \& Schuster 1999).

The analytical formula for the resolution limit $\Delta x$ can be derived by assuming the scatterer to be at $\mathbf{x}_{o}=\left(x_{o}, z_{o}\right)$ and the geophone at $\mathbf{g}=(0,0)$, so that the two-way zero-offset reflection traveltime is $\tau_{g x g}=2 \sqrt{x_{o}^{2}+z_{o}^{2}} / c$. This traveltime can be differentiated $\mathrm{w} / \mathrm{r}$ to the scatterer's lateral coordinate to give $d \tau_{g x g} / d x_{o}=2 x_{o} / c \sqrt{x_{o}^{2}+z_{o}^{2}}$. Rearranging gives

$d x_{o}=\frac{c d \tau_{g x g} \sqrt{x_{o}^{2}+z_{o}^{2}}}{2 x_{o}}$.

Setting $d \tau_{\text {gxg }}$ to be the dominant period $T$ of the source wavelet yields the formula for horizontal resolution $\Delta x$ in the migration image at $\mathbf{x}_{o}$ :

$$
\begin{aligned}
\Delta x & =\frac{\lambda}{2} \frac{\sqrt{x_{o}^{2}+z_{o}^{2}}}{x_{o}}, \\
& \approx \frac{\lambda}{2} \frac{z_{o}}{L},
\end{aligned}
$$

where the last equation follows from the far-field approximation for $z_{o} \gg L$ and a small enough wavelength. Here, the best horizontal resolution $\Delta x$ is sought so we set $x_{o} \rightarrow L$ for the maximum offset between a trace and a scatterer centred below the middle of the array in Fig. 2. To be consistent with the resolution associated with the first-Fresnel zone, $d \tau_{g x g}$ should be set to $\mathrm{T} / 2$ rather than $\mathrm{T}$. However, we will use a conservative value of $d \tau \rightarrow T$ that is consistent with the diagrams in Fig. 2.
Eq. (4) says that $\Delta x$ is linearly proportional to the depth of the scatterer $z_{o}$ and the dominant wavelength $\lambda$, and is inversely proportional to the aperture width $2 L$. This implies that wider apertures, shallower point scatterers, and a wider source bandwidth improve the lateral resolution of the point-scatterer's image. For vertical resolution the trace directly above the scatterer is used to give the best vertical resolution

$\Delta z=\lambda / 2$,

which is the radial width of the migration doughnut.

Eqs (4) and (5) characterize how much the time-domain wavelet gets stretched along the spatial coordinates of the migration image. This mapping is known as migration stretch, which is equivalent to the resolution limits along different coordinate directions (Berkhout 1984; Safar 1985; Chen \& Schuster 1999).

\subsection{Migration stretch limits for acausal lossy media}

The above analysis assumed a lossless medium. However, a lossy medium will give rise to a decay of higher frequencies so that the dominant period of the diffraction event increases with offset. This will lead to wavelet broadening and even greater stretching in the migration image, so the resolution limits need to be modified for attenuation.

For a constant $Q$ and constant velocity independent of frequency, the zero-offset band-limited response of a single scatterer located at $\mathbf{x}_{o}$ is given by

$$
\begin{aligned}
d(\mathbf{g}, t) & =\frac{1}{2 \pi} \int_{-\omega_{o}}^{\omega_{o}} \mathrm{e}^{-(i \omega \alpha+|\omega| \beta)} \mathrm{d} \omega, \\
& =\frac{1}{2 \pi} \int_{0}^{\omega_{o}}\left[\mathrm{e}^{-\omega(i \alpha+\beta)}+\mathrm{e}^{\omega(i \alpha-\beta)}\right] \mathrm{d} \omega, \\
& =\frac{1}{2 \pi}\left[\left.\frac{\mathrm{e}^{-\omega(i \alpha+\beta)}}{-i \alpha-\beta}\right|_{0} ^{\omega_{o}}+\left.\frac{\mathrm{e}^{\omega(i \alpha-\beta)}}{i \alpha-\beta}\right|_{0} ^{\omega_{o}}\right], \\
& =\frac{1}{2 \pi}\left[\frac{\mathrm{e}^{-\omega_{o}(i \alpha+\beta)}}{-i \alpha-\beta}+\frac{1}{i \alpha+\beta}+\frac{\mathrm{e}^{\omega_{o}(i \alpha-\beta)}}{i \alpha-\beta}-\frac{1}{i \alpha-\beta}\right], \\
& =\frac{1}{\pi\left(\alpha^{2}+\beta^{2}\right)}\left[\mathrm{e}^{-\omega_{o} \beta}\left\{\alpha \sin \left(\omega_{o} \alpha\right)-\beta \cos \left(\omega_{o} \alpha\right)\right\}+\beta\right],
\end{aligned}
$$

where $\alpha=2 r / c-t, \beta=r / c Q, \omega_{o}=2 \pi / T_{o}$ is the maximum angular frequency, $\mathbf{g}=\left(x_{g}, 0\right)$ denotes the location of the 
(a) $\mathrm{d}(\mathrm{g}, \mathrm{t})$ vs Time for $\mathrm{Q}=15$

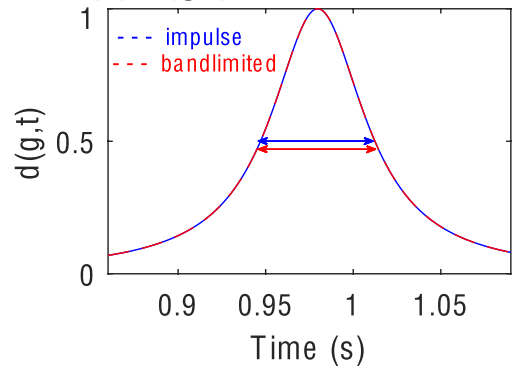

(c) $\Delta_{t}$ vs $\mathbf{Q}$

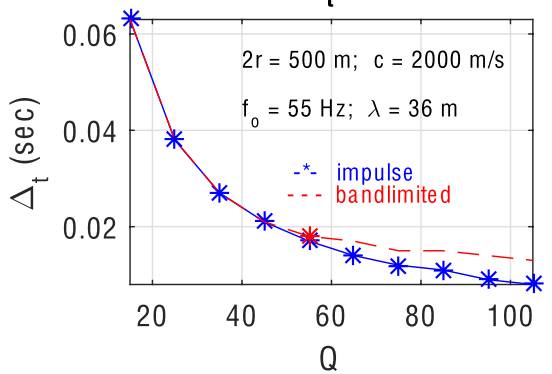

(b) $\mathrm{d}(\mathrm{g}, \mathrm{t})$ vs Time for $\mathrm{Q}=105$

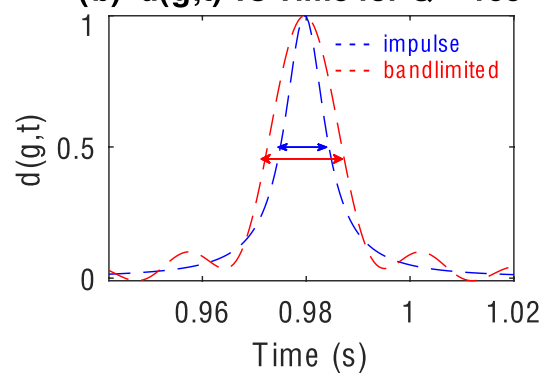

(d) $T^{\text {atten }} / T_{0}$ vs $Q$

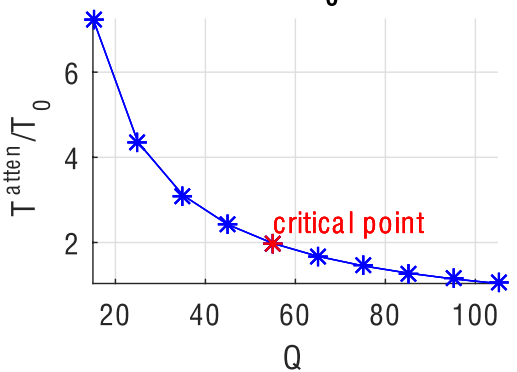

Figure 3. Plots of normalized $d$ (g, $t$ ) against time for (a) $Q=15$ and (b) $Q=105$, where the red band-limited (see eq. 6) and blue impulse (see eq. 9) source wavelets propagated $500 \mathrm{~m}$ (about 15 wavelengths at the highest frequency) from the point source in a homogeneous attenuative medium. The temporal wavelet widths $\Delta_{t}$ (double-sided arrows) at the half-amplitude of the impulsive (blue) and band-limited (red) wavelets and the $T^{\text {atten }} / T_{o}$ ratios are plotted in (c) and (d), respectively, against different $Q$ values. The agreement between the impulse and band-limited values of $\Delta_{t}$ is excellent for $Q \leq 50$ in (c) when $T^{\text {atten }} / T_{o} \geq 2$ in (d), where the red star denotes the critical value of $T^{\text {atten }} / T_{o}=2$.

geophone on the horizontal recording plane at $z=0, r=\left|\mathbf{g}-\mathbf{x}_{o}\right|$ is the distance between the scatterer and the geophone, $e^{-\omega r /(c Q)}$ accounts for attenuation in the zero-offset reflection trace and the strength of attenuation is associated with the constant $1 / Q$. It is assumed that the geometric spreading term is compensated here by an illumination compensation operation. The above constant $Q$ and constant velocity model is simple, but it leads to acausal arrivals (Kjartansson 1979).

Eq. (6) represents the zero-offset data that are to be migrated, and examples of the associated spectra and their seismograms are shown in Fig. 1. In this case, the green arrivals have propagated around 30 wavelengths from the source to the far-field receiver. Here, $\frac{\partial w(t)}{\partial t}$ is the source wavelet, where $w(t)$ is a zero-phase wavelet with a Gaussian spectrum peaked at $30 \mathrm{~Hz}$. Note, much of the propagation energy in the acausal far-field trace in (b) arrives before the expected propagation time denoted by the blue vertical bar.

\subsubsection{Wavelet width and large $Q$}

For a nearly lossless medium where $Q \rightarrow$ large, the exponent in the integrand of eq. (6) becomes $\lim _{Q \rightarrow \operatorname{large}} \mathrm{e}^{-|\omega| r / c Q} \rightarrow 1$ so the direct-wave arrival can be approximated by a time-shifted sinc function

$\lim _{Q \rightarrow \operatorname{large}} d(\mathbf{g}, t) \approx \frac{\sin \omega_{o} \alpha}{\pi \alpha}$,

where the width of the main lobe at the half-amplitude value is proportional to the minimum period $0.6 T_{o}=0.6 / f_{o}$ (Note, the width of the sinc function at half the peak amplitude is $0.6 T_{o}$ ). This says that for a nearly lossless medium the minimum period $T_{o}$, not the quality factor Q, determines the wavelet width.

As an example, the red signal in Fig. 3(b) depicts the band-limited arrival for $Q=105$, a maximum source frequency $f_{o}=55 \mathrm{~Hz}$ and a propagation distance $2 r=1 \mathrm{~km}$. The more general condition that drives the exponential $e^{-\omega_{o} \beta}$ to unity is $\omega_{o} \beta=\omega_{o} r / c Q \approx 0$, which can be expressed as

$$
\pi \overbrace{T^{\text {atten }}}^{2 r / c Q} / T_{0} \approx 0 .
$$

As we will be shown in the next section, $T^{\text {atten }}=2 r / c Q$ is the wavelet width (see eq. 13), or attenuation stretch, associated with a wideband direct wave that has propagated the distance $2 r$ in a homogeneous lossy medium. Therefore, eq. (8) says that the large $Q$ condition implies that the attenuation stretch must be much smaller than the minimum period of the source wavelet.

\subsubsection{Wavelet width and small $Q$}

Highly attenuative media implies $\lim _{Q \rightarrow \text { small }} \mathrm{e}^{-\omega_{o} r / c Q} \rightarrow 0$ so that eq. (6) becomes

$$
\begin{aligned}
\lim _{Q \rightarrow \text { small }} d(\mathbf{g}, t) & \approx \frac{\beta}{\pi\left(\alpha^{2}+\beta^{2}\right)}, \\
& =\frac{r /(c Q)}{\pi\left[\{r /(c Q)\}^{2}+(2 r / c-t)^{2}\right]},
\end{aligned}
$$

where the peak amplitude

$d\left(\mathbf{g}, t^{\text {peak }}=2 r / c\right)=\frac{c Q}{\pi r}$,

is recorded at the peak time $t^{\text {peak }}=2 r / c$. Instead of a sinc-like arrival, $d(\mathbf{g}, t)$ is characterized by the time-shifted Gaussian-like arrival in Fig. 3(a) (see section 3.7.8 in Stein \& Wysession 2003). Eq. (9) is also true when $\omega_{o}$ is large enough, so we will sometimes denote $d(\mathbf{g}, t)$ here as the wideband signal.

To determine the wavelet stretch in the migration image, we define the attenuation stretch $T^{\text {atten }}$ as the width of the arrivals, in either Fig. 3(a) or (b), where the amplitude is half that at the peak amplitude. In this case the ratio of the trace amplitudes 
$d(\mathbf{g}, t) / d\left(\mathbf{g}, t^{\text {peak }}\right)$ can be set equal to 0.5 at the half-amplitude time $t=t_{0.5}$, that is,

$\frac{d\left(\mathbf{g}, t_{0.5}\right)}{d\left(\mathbf{g}, t^{\text {peak }}=2 r / c\right)}=\frac{\pi r^{2} /(c Q)}{\pi c Q\left[\{r /(c Q)\}^{2}+\left(2 r / c-t_{0.5}\right)^{2}\right]}=0.5$.

Solving for the half-amplitude time $t_{0.5}$ in the above equation gives

$t_{0.5}=\frac{2 r}{c}-\frac{r}{c Q}$,

so that the attenuation stretch is

$T^{\text {atten }}=2\left(t^{\text {peak }}-t_{0.5}\right)=\frac{2 r}{c Q}=2 \beta$,

where $t^{\text {peak }}$ is defined in eq. (10). Unlike the width of the sinc function in eq. (7), $T^{\text {atten }}$ is independent of the maximum angular frequency $\omega_{o}$.

The accuracy of the wideband approximation in eq. (9) is tested in Figs 3(a) and (b), where the wideband blue signals are computed by eq. (9) and the red band-limited signals are computed from eq. (6) for different $Q$ values in a homogeneous medium. In this example, the maximum frequency of the band-limited wavelet is $\omega_{o} / 2 \pi=55 \mathrm{~Hz}$ and the propagation distance is $500 \mathrm{~m}$. The temporal widths at the half-amplitude of the impulsive (blue) and band-limited (red) wavelets are plotted in (c) against Q for the source-receiver offset of $500 \mathrm{~m}$. For this example, (c) and (d) suggest that the width of the wavelet predicted by $T^{\text {atten }}=2 r / c Q$ in eq. (13) is a reliable prediction when it is more twice the value of $T_{o}$ (see the red critical points in Figs $3 \mathrm{c}$ and d).

\subsubsection{Resolution limits}

The previous sections demonstrated that for large $Q$ the wavelet width of a band-limited direct arrival is equal to the smallest period $T_{o}$, while it is $T^{\text {atten }}=2 r / c Q$ for small $Q$. The more general formula for $T^{\text {atten }}$ is derived in Appendix A (see eq. A8), but it is unnecessarily complex for estimating simple resolution limits. To avoid this complexity, we use either the large $\omega_{o} r / c Q$ or small $\omega_{o} r / c Q$ approximations, analogous to the near- or far-field approximations in a lossless medium. Therefore, we define the effective wavelet stretch as

$\tilde{T}^{\text {atten }}=\left\{\begin{array}{ll}2 r / c Q & \text { for } \omega_{o} r / c Q \gg 0 \\ 0.6 T_{o} & \text { for } \epsilon>\omega_{o} r / c Q\end{array}\right.$,

where $1 \gg \epsilon>0$. Multiplying $\tilde{T}^{\text {atten }}$ by the velocity $c$ gives the effective wavelength

$\tilde{\lambda}=\left\{\begin{array}{ll}2 r / Q & \text { for } \omega_{o} r / c Q \gg 0 \\ 0.6 c T_{o} & \text { for } \epsilon>\omega_{o} r / c Q\end{array}\right.$,

and inserting eq. (15) into eq. (4) gives the horizontal resolution limits:

$\tilde{\Delta} x^{\text {lossy }}=\left\{\begin{array}{ll}\Delta x^{\text {lossy }}:=z_{o}^{2} / Q L & \text { for } \omega_{o} r / c Q \gg 0 \\ \Delta x:=0.6 c T_{o} z_{o} / 2 L & \text { for } \epsilon>\omega_{o} r / c Q\end{array}\right.$,

where $r \approx z_{o}$ and $\Delta x=\frac{0.6 \lambda^{\min } z_{o}}{2 L}$ is the lossless resolution formula. Therefore, lossy horizontal resolution worsens linearly with increasing $1 / Q$ and quadratically with increasing depth $z_{o}$ of the scatterer.
Note, the resolution limits depend on our arbitrary choice for what constitutes an effective width of the wavelet. The effective width of the wavelet is $T_{o}$ for a sinc function if it is defined to be the distance between adjacent zero crossings of the main lobe. In this case $\Delta x$ in eq. (16) will become $c T_{o} z_{o} / 2 L$ and be in agreement with that in eq. (4).

To compute the vertical-resolution limit $\Delta z^{\text {lossy }}$ we assume a trace directly above the scatterer so that

$\Delta z^{\text {lossy }}=\frac{c T^{\text {atten }}}{2}=\frac{z_{o}}{Q}$.

Unlike the lossless limit $\Delta z=\lambda / 2$ in eq. (5), the lossy vertical resolution gets worse with increasing depth of the scatterer and decreasing values of $Q$. Simply put, loss in the resolution formulae is accounted for by replacing $\lambda$ with $z / Q$.

\subsection{Migration stretch limits for causal lossy media}

The problem with the constant $Q$ and frequency-independent velocity model is that its impulse response is non-causal. Therefore, an attenuation model is required that enforces causality so that no signals arrive earlier than allowed by the actual propagation velocity. As an example, compare the acausal green event in Fig. 1(b) to the causal one Fig. 1(d).

To enforce causality in the impulse response, Kjartansson (1979) assumed an attenuation model where there is a linear and causal relation between stress $\sigma(t)$ and strain $\epsilon(t)$ :

$\sigma(t)=\kappa(t) \star \epsilon(t) ; \epsilon(t)=s(t) \star \sigma(t)$,

$\epsilon(t)=\sigma(t)=0 \quad$ for $t<0$,

where $\star$ indicates temporal convolution. Here, $\kappa(t)$ and $s(t)$ are the time-dependent bulk modulus and compliance that honour causality so that the stress does not begin before the excitation of strain, and vice versa, that is, $\kappa(t)=s(t)=0$ for $t<0$. Therefore, the stressstrain relations can be combined with Newton's equilibrium law to give the 1D lossy wave equation in the frequency domain:

$\left[\frac{\mathrm{d}^{2}}{\mathrm{~d} x^{2}}+\frac{\omega^{2}}{c(\omega)^{2}}\right] u(x, \omega)=0$,

where

$u(x, \omega)=e^{i \frac{\omega}{c(\omega)} x}$ and $c(\omega)=\sqrt{\bar{\kappa}(\omega) / \rho}$,

are the particle displacement and dispersive propagation velocity in a homogeneous medium, the density is $\rho$, and the complex frequency-domain bulk modulus is $\bar{\kappa}(\omega)$. Here, $x=2 r$ is the propagation distance.

Frequency-independent $Q$ implies that the loss per cycle is independent of the time scale of oscillation. In this case, Kjartansson (1979) claims that the traveltime $T$ and the pulse width $T^{\text {atten }}$ can be related to one another by

$T^{\text {atten }}=\frac{C(Q)}{Q} T \propto \frac{C(Q)}{Q}\left(\frac{2 r}{c_{o}}\right)^{\bar{\beta}}$,

where

$\bar{\beta} \approx 1+\frac{1}{\pi Q}$,

and $C(Q)$ is a constant for $Q>20$. Here, the two-way traveltime $T=\left(2 r / c_{o}\right)^{\bar{\beta}}$ is the apparent propagation time from the source to the scatterer and back to the geophone.

Multiplying eq. (21) by $c_{o}$ so that $T^{\text {atten }} c_{o}=\lambda$ and inserting $\lambda$ into eq. (4) gives the horizontal-resolution limit in a lossy-causal 
(a) ZO Data: $Q=200$

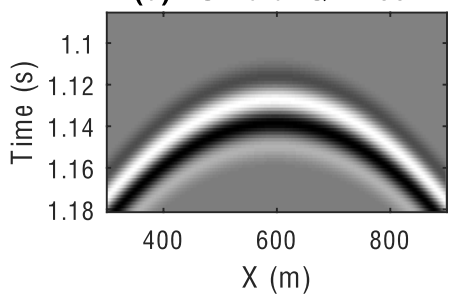

(c) ZO Data: $Q=50$

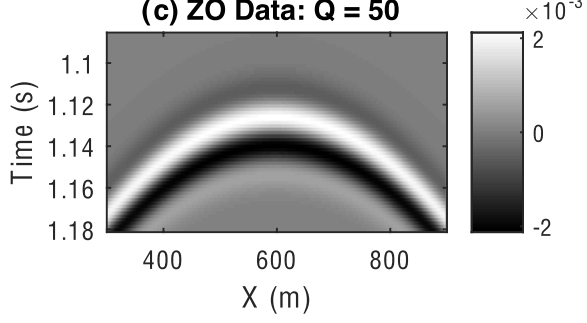

(e) ZO Data: $Q=20$

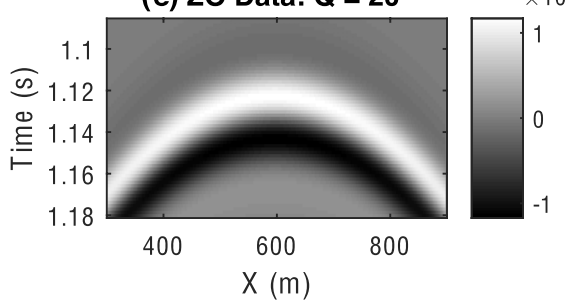

(b) ZO Migration: $Q=\mathbf{2 0 0}$

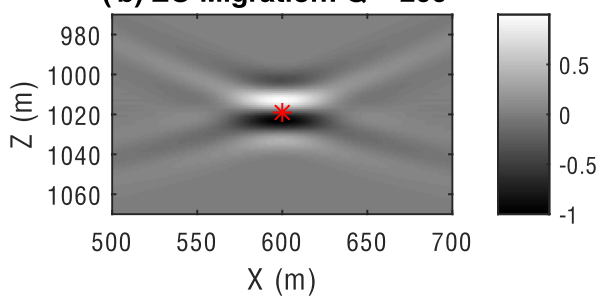

(d) ZO Migration: $Q=50$

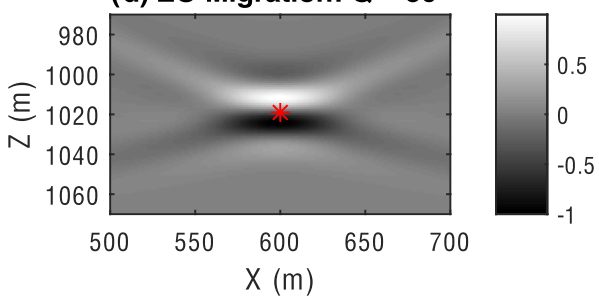

(f) ZO Migration: $Q=20$

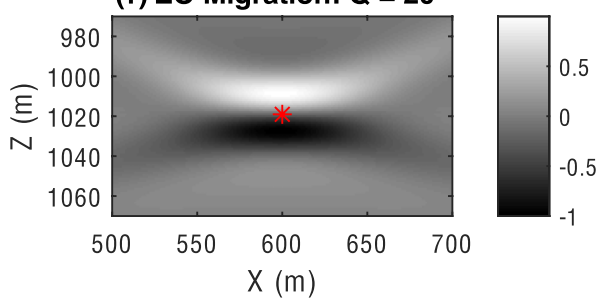

Figure 4. Left column displays zero-offset traces and right-column depicts the corresponding ZO migration images for a point source (red star) buried at a depth $1.02 \mathrm{~km}$ and centred below a $0.6 \mathrm{~km}$ line of geophones. Each row of images is computed for a homogeneous model with a velocity of $0.9 \mathrm{~km} \mathrm{~s}{ }^{-1}$ and a different $Q$ value in the model.

medium:

$\Delta x_{\text {causal }}^{\text {lossy }} \propto C(Q) \frac{2^{\bar{\beta}-1} z_{o}^{\bar{\beta}+1}}{c_{o}^{\bar{\beta}-1} Q L}$,

where Kjartansson (1979) shows that $C(Q) \approx 0.5$ for $Q>20$. For $Q>20, \bar{\beta} \approx 1$ and $\Delta x_{\text {causal }}^{\text {loss }}$ becomes worse quadratically with increasing depth $z_{o}$ of the scatterer, which is similar to the acausallossy resolution limit in eq. (16). It is straightforward to show that $\Delta z_{\text {causal }}^{\text {lossy }}$ gets worse linearly with increasing depth of the scatterer, similar to $\Delta z^{\text {lossy }}$ in eq. (5).

As an example, the spectra and seismograms for the causal Q model are shown in Figs 1(c) and (d), respectively. They are similar to those for the acausal Q model in Figs 1(a) and (b), except now much of the energy arrives past the propagation time denoted by the blue vertical bar. If the $\mathrm{Q}$ distribution is known, then there are schemes for partial attenuation compensation in migration images (Wang \& Guo 2004; Dutta \& Schuster 2014, 2016; Dutta 2016).

\subsection{Extension to linearized waveform inversion}

The migration equation is proportional to the gradient of the waveform misfit function (Lailly 1984; Mora 1989) in waveform inversion (see Appendix B). Therefore, any velocity image obtained from linearized waveform inversion in a lossy medium will have similar resolution limits $\Delta_{x}^{\text {lossy }}$ and $\Delta_{z}^{\text {lossy }}$ associated with a migration image. This assumes that only primary reflections are inverted in the waveform inversion algorithm and that loss adjustments are made for the non-zero offset between the source and receiver.

We note that the waveform inversion algorithm has two types of gradients: the tomographic update (Mora 1989; Xu et al. 2012) of velocities along cigar-like and rabbit-ear wave paths, and the other is for updating velocities along the migration ellipses (Huang \& Schuster 2014). The resolution formulae derived in the previous sections are those for migration ellipses in a lossy medium. To derive the resolution limits for the tomographic operator we recall the tomographic resolution limit $\Delta_{\perp}$ perpendicular to the cigar-like gradient (Huang \& Schuster 2014) as

$\Delta_{\perp} \propto \sqrt{\lambda 2 r}$,

where $2 r$ is the distance between the source and geophone in a homogeneous medium with dominant wavelength $\lambda=c T$ and dominant period $T$. For lossy media the wavelength in eq. (24) is replaced by the effective wavelength in eq. (15) to give the lossy resolution limit

$\Delta_{\perp}^{\text {lossy }} \propto \sqrt{\frac{4 r^{2}}{Q}}$.

This says that $\Delta_{\perp}^{\text {lossy }}$ worsens as $1 / \sqrt{Q}$ for the tomographic gradient compared to $\Delta_{x}^{\text {lossy }}, \Delta_{z}^{\text {lossy }} \propto 1 / Q$ for the migration gradient.

\section{NUMERICAL RESULTS}

A homogeneous velocity model with a point source at the depth of $1.12 \mathrm{~km}$ is used to assess the migration resolution limits for lossy media. Only the results for the acausal Q model are provided because the causal Q results are similar. Here, the source is assumed to be buried at the scatterer's location, and the geophones on the surface record the resulting seismic waves propagating through the model with velocity $0.9 \mathrm{~km} \mathrm{~s}^{-1}$. This resembles the exploding reflector model for migration of post-stack data.

The left column of Fig. 4 shows the zero-offset data generated for models with (a) $Q=200$, (c) $Q=50$, (e) $Q=20$ and a 
(a) Horizontal Migration Profile

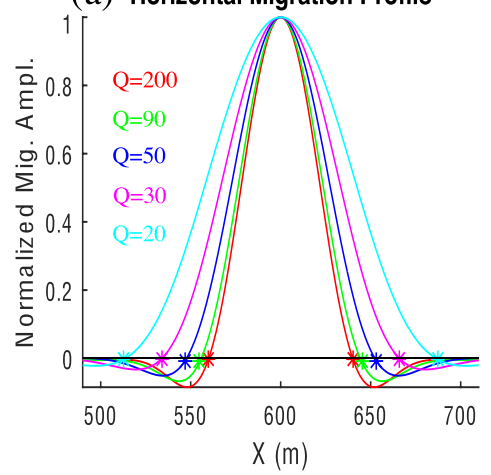

(c)

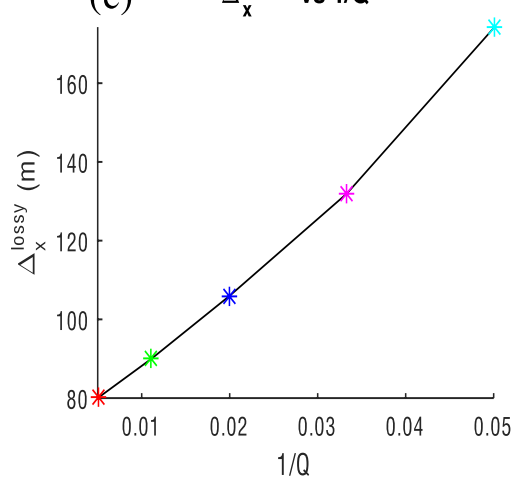

(b) Vertical Migration Profile

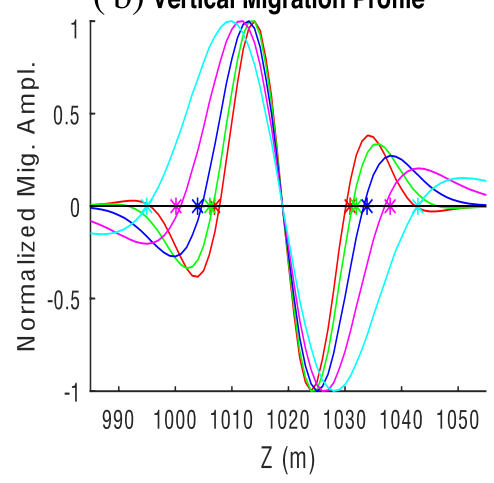

(d)

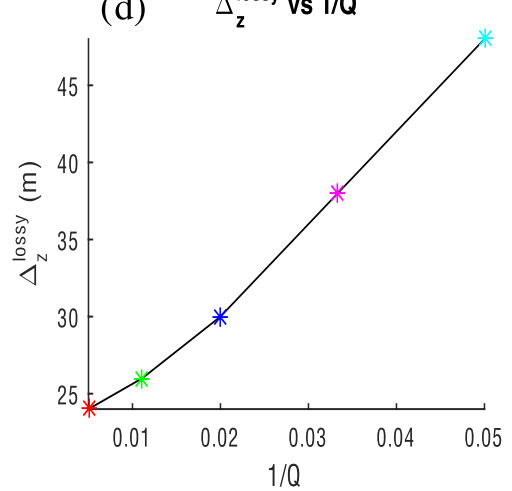

Figure 5. Migration profiles along the (a) horizontal and (b) vertical slices of the migration image centred at the location of the buried point source (i.e. scatterer). The resolution limits for (c) $\Delta_{x}^{\text {lossy }}$ (distance between the starred zero-crossings of the same colour in (a)) and (d) $\Delta_{z}^{\text {lossy }}$ (distance between the starred zero-crossings of the same colour in (b)) are for the migration images on the right-side column of Fig. 4 for different values of $Q$.

source wavelet with a Gaussian-like spectrum peaked at $30 \mathrm{~Hz}$. The acausal seismograms are migrated with diffraction stack migration (see eq. 1) and the resulting migration images are shown on the right column of Fig. 4. It can be qualitatively seen that both the verticaland horizontal-resolution limits of the migration images decrease with increasing values of $1 / Q$ as the theory predicts.

The top row of Fig. 5 depicts the zoom views of the migration profiles along the (a) horizontal and (b) vertical axes, centred at the position of the buried point source. In each plot, there are 5 profiles associated with the 5 different Q values listed in Fig. 5(a). Defining $\Delta_{x}^{\text {lossy }}$ to be the distance between adjacent zero crossings that neighbour the main lobe in Fig. 5(a) and plotting $\Delta_{x}^{\text {lossy }}$ against $1 / Q$ gives the curve shown in Fig. 5(c). We observe that $\Delta_{x}^{\text {lossy }}$ increases linearly with $1 / Q$, as predicted by eq. (16). Similarly, the migration profiles along the vertical axis are displayed in Fig. 5(b), and the associated values of $\Delta_{z}^{\text {lossy }}$ are plotted against $1 / Q$ in Fig. 5(d). This curve is consistent with the linear relationship between $\Delta_{z}^{\text {lossy }}$ and $1 / Q$ predicted by eq. (17).

To numerically assess the resolutions limits as a function of scatterer depth $z_{o}$, the ZO data in Fig. 6(a) are computed for the lossy model with five point sources indicated by the red stars in the Fig. 6(b) migration images. In this case the background velocity model has a velocity of $c=0.9 \mathrm{~km} \mathrm{~s}^{-1}$ and $Q=30$. The corresponding horizontal and vertical migration profiles are displayed in the top row of Fig. 7. The distance between zero-crossings with the same colour are taken to be the resolution limits, and $\Delta x^{\text {lossy }}$ is plotted against $z_{o}^{2}$ in Fig. 7(c). As predicted by formula 16, $\Delta x^{\text {lossy }}$ worsens quadratically with the depth of the scatterer. The vertical- resolution limit $\Delta z^{\text {lossy }}$ in Fig. 7(d) only worsens linearly with depth of the scatterer, as predicted by eq. (17).

\section{CONCLUSIONS AND DISCUSSION}

The migration resolution limits are derived in the far-field approximation for a point scatterer in a lossy homogeneous medium for both the causal and acausal attenuation models. Analysis shows that the horizontal-resolution limits $\Delta x^{\text {lossy }} \propto z_{o}^{2} / Q L$ and $\Delta x_{\text {causal }}^{\text {loss }}$ worsen quadratically with increasing depth compared to $\Delta x \propto \lambda z_{o} / L$ for a lossless medium. Also, the vertical-resolution limits $\Delta z^{\text {lossy }}$ and $\Delta z_{\text {causal }}^{\text {lossy }}$ grow linearly with increasing depth of the scatterer $z_{o}$. In all cases, the resolution limits for a highly lossy medium can be approximated by replacing the actual wavelength $\lambda$ in $\Delta_{x}$ and $\Delta_{z}$ by the lossy wavelength $z_{o} / Q$. For both the causal and acausal models, the resolution limits worsen with increasing values of $1 / Q$. Since the migration image is proportional to the gradient of the misfit function, these resolution limits are also applicable to those for linearized waveform inversion.

A practical application of the above methodology is to invert for the $\mathrm{Q}$ distribution from the wavelet stretch in the data or the migration images. For example, eq. (13) describes, for a given velocity, the dominant period in the wavelet as a function of distance travelled and the Q in the medium. Similar to the NMO formula that describes traveltime as a function of velocity and distant travelled (Yilmaz 2001), a Q-NMO formula can be derived that describes how the period of reflections in a CMP gather changes with offset. Analogous to velocity semblance analysis applied to a common 
(a) ZO Data

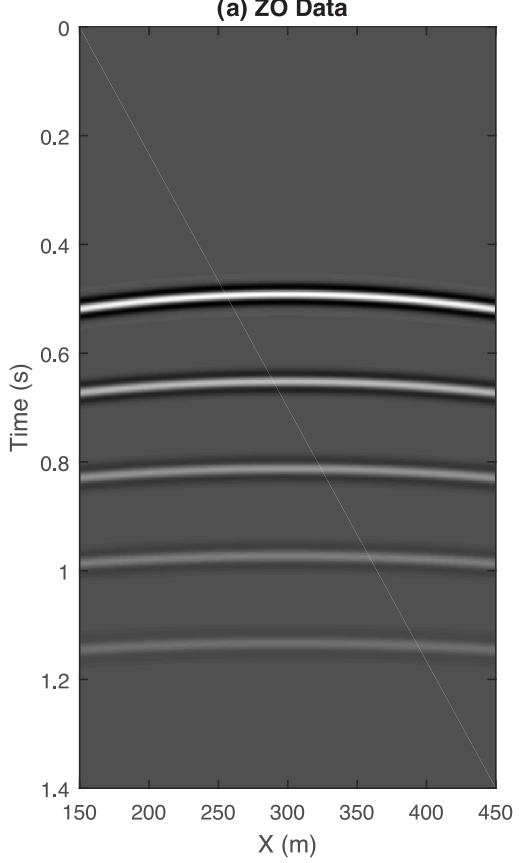

(b) Migration Image

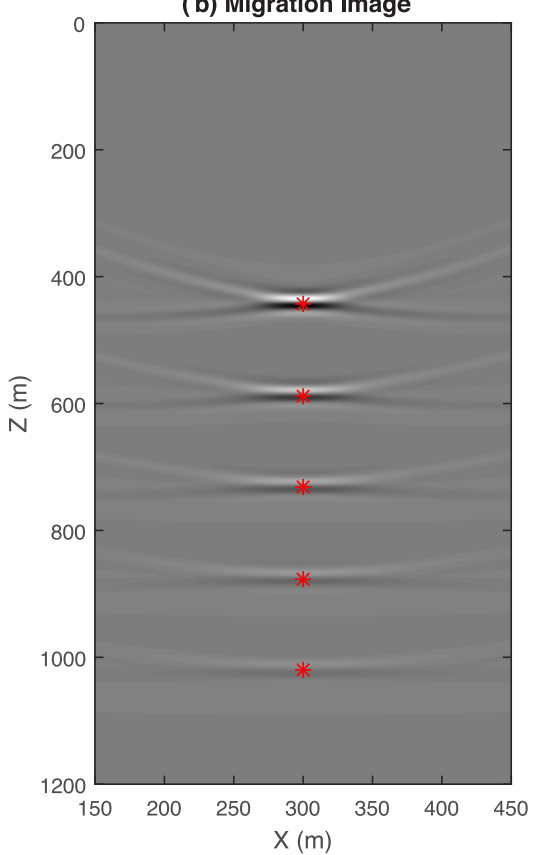

Figure 6. (a) ZO data and (b) migration image for a lossy model with $Q=30$ and five point sources located at the red stars.

(a) Horizontal Migration Profile

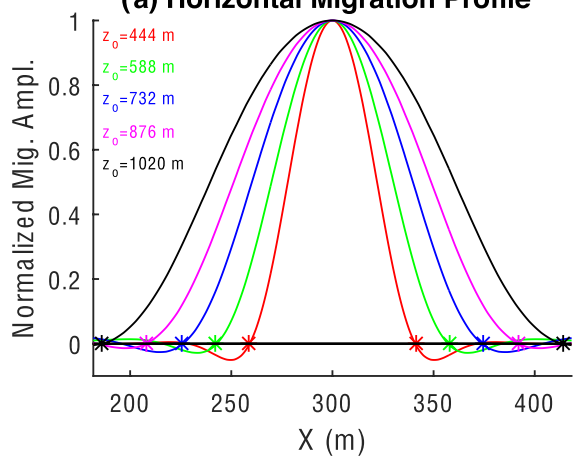

(c) $\Delta_{x}^{\text {lossy }}$ vs $z_{0}^{2}$

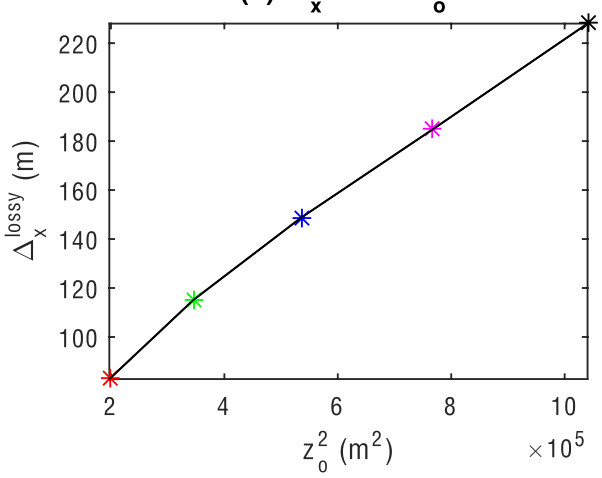

(b) Vertical Migration Profile

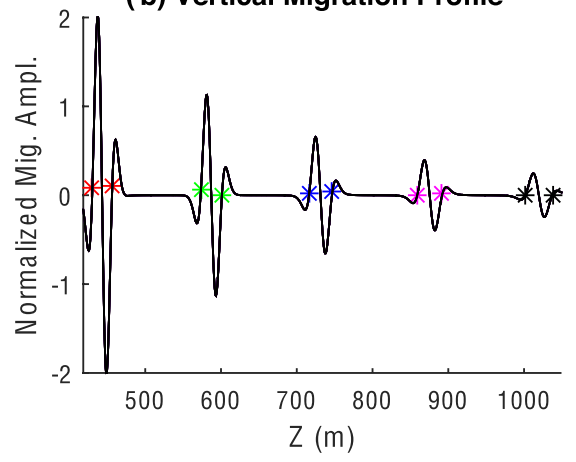

(d) $\Delta_{z}^{\text {lossy }}$ vs zo

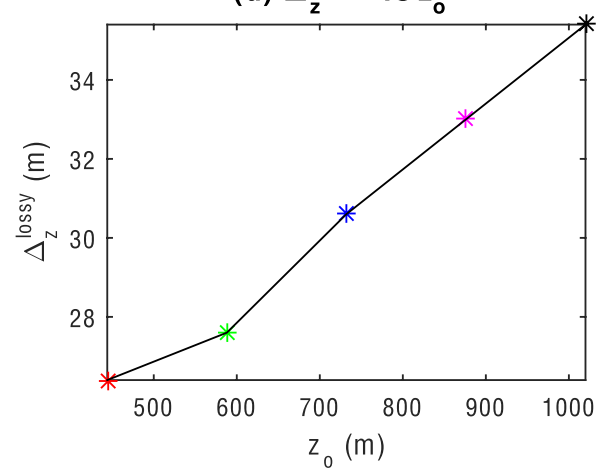

Figure 7. (a) Horizontal and (b) vertical migration profiles for the Fig. 6 migration image, where the horizontal profiles are centred at the five different scatterers. Figs (c) and (d) depict the associated resolution limits, which are taken to be the distances between zero-crossings of the same colour in the top row of plots. The plots validate the theoretical predictions in eqs (16) and (17), where (c) $\Delta_{x}^{\text {lossy }} \propto z_{o}^{2}$ and (d) $\Delta_{z}^{\text {lossy }} \propto z_{o}$, where $z_{o}$ is the depth of the scatterer.

midpoint gather (Yilmaz 2001), a Q-semblance analysis can be used to invert for $Q(z)$ from the observed changes in the reflection's period with offset. In the migration image domain, the migration stretch formula can be used to estimate the Q distribution from the picked stretched values $\Delta z$ in the common image gathers. This is analogous to migration velocity analysis in the common image gather domain (Yilmaz 2001), except the Q distribution is estimated.

\section{ACKNOWLEDGEMENTS}

The research reported in this publication was supported by the King Abdullah University of Science and Technology (KAUST), Saudi Arabia. We are grateful to the sponsors of the Center for Subsurface Imaging and Modeling (CSIM) Consortium for their financial support. We also thank KAUST for providing funding by 
the CRG grant OCRF-14 2014-CRG3-2300. We thank the Editor Dr Lapo Boschi and the two anonymous reviewers for their helpful comments and suggestions.

\section{REFERENCES}

Berkhout, A.J., 1984. Seismic Resolution: A Quantitative Analysis of Resolving Power of Acoustical Echo Techniques, Geophysical Press.

Berkhout, A.J. \& Van Wulfften, E., 1979. Migration in terms of spatial deconvolution, Geophys. Prospect., 27, 261-291.

Beylkin, G., Oristaglio, M. \& Miller, D., 1985. Spatial resolution of migration algorithms, in Acoustical Imaging, pp. 155-168, eds Berkhout, A. J., Ridder, J. \& van der Wal, L.F., Springer.

Bleistein, N., 1987. On the imaging of reflectors in the earth, Geophysics, 52, 931-942.

Carrion, P.M., Sato, H.K. \& Buono, A.V., 1991. Wavefront set analysis of limited aperture migration sections, Geophysics, 56, 778-784.

Chen, J. \& Schuster, G.T., 1999. Resolution limits of migrated images, Geophysics, 64, 1046-1053.

Cohen, J.K., Hagin, E.C. \& Bleistein, N., 1986. Three-dimensional Born inversion with an arbitrary reference, Geophysics, 51, 1552-1558.

Claerbout, J.F., 1992. Earth Soundings Analysis: Processing vs Inversion, Blackwell Scientific Inc.

Dahlen, F.A., 2004. Resolution limit of traveltime tomography, Geophys. J. Int., 157, 315-331.

Dutta, G., 2016, Skeletonized wave-equation inversion for Q, in 2016 SEG Annual Meeting Abstract, pp. 3618-3623.

Dutta, G. \& Schuster, G.T., 2014. Attenuation compensation for leastsquares reverse time migration using the viscoacoustic-wave equation, Geophysics, 79, S251-S262.

Dutta, G. \& Schuster, G.T., 2016. Wave-equation Q tomography, Geophysics, 81, R471-R484.

French, W., 1974. Two-dimensional and three-dimensional migration of model-experiment reflection profiles, Geophysics, 39, 265-277.

Huang, Y. \& Schuster, G.T., 2014. Resolution limits for wave equation imaging, J. Appl. Geophys., 107, 137-148.

Kjartansson, E., 1979. Constant Q-Wave propagation and attenuation, Geophysics, 84, 4737-4748.

Lailly, P., 1984. Migration methods: partial but efficient solutions to the seismic inverse problem, in Inverse Problems of Acoustic and Elastic Waves, p. 184, ed. Santosa, F., SIAM.

Li, J., Dutta, G. \& Schuster, G.T., 2017. Wave-equation Qs inversion of skeletonized surface waves, Geophys. J. Int., 209(2) 979-991.

Luo, Y. \& Schuster, G.T., 1991. Wave equation inversion of skeletalized geophysical data, Geophys. J. Int., 105, 289-294.

Mora, P., 1989. Inversion=migration+tomography, Parallel Comput., 54, 78-101.

Safar, M.H., 1985. On the lateral resolution achieved by Kirchhoff migration, Geophysics, 50, 1091-1099.

Sheng, J. \& Schuster, G.T., 2003. Finite-frequency resolution limits of wave path traveltime tomography for smoothly varying velocity models, Geophys. J. Int., 152, 669-676.

Stein, S. \& Wysession, S., 2003. An Introduction to Seismology: Earthquakes and Earth Structure, Blackwell Publishing.

Wang, Y. \& Guo, J., 2004. Seismic migration with inverse $Q$ filtering, Geophys. Res. Lett., 31, L21608, doi:10.1029/2004GL020525.

Williamson, P.R. \& Worthington, M.H., 1993. Resolution limits in ray tomography due to wave behavior: numerical experiments, Geophysics, 58, 727-735.

Witten, A. \& Molyneux, J.E., 1988. Geophysical imaging with arbitrary source illumination, IEEE Trans. Geosci. Remote Sens., 26, 409-419.

Wu, R.S. \& Toksoz, M.N., 1987. Diffraction tomography and multisource holography applied to seismic imaging, Geophysics, 52, 11-23.

Xu, S., Wang, D., Chen, F., Zhang, Y. \& Lambáre, G., 2012. Full waveform inversion for reflected seismic data, in 74th Annual International Conference and Exhibition, EAGE, Extended Abstracts, W024.

Yilmaz, O., 2001. Seismic Data Analysis, SEG Press Book.

\section{APPENDIX A: FORMULA FOR BAND-LIMITED WAVELET STRETCH}

The formula for the attenuation stretch $T^{\text {atten }}$ in eq. (13) is for an impulsive source with a wideband spectrum. However, this formula will be much more complicated for the band-limited signal described by eq. (6). Here, $d(\mathbf{g}, t)$ is symmetrical and has its peak amplitude

$d\left(\mathbf{g}, t^{\text {peak }}\right)=\frac{1}{\pi \beta}\left(1-e^{-\omega_{o} \beta}\right)$,

at $t^{\text {peak }}=2 r / c$ for any specified distance $r$. Eq. (A1) is derived by inserting $t=2 r / c$ into eq. (6) so that $\alpha=0$.

The time $t_{0.5}$ in which the amplitude of $d(\mathbf{g}, t)$ falls to $1 / 2$ its peak value can be found by solving

$$
\begin{aligned}
\frac{d\left(\mathbf{g}, t_{0.5}\right)}{d\left(\mathbf{g}, t^{\text {peak }}\right)}= & \frac{\beta}{\left(\alpha^{2}+\beta^{2}\right)} \\
& \times \frac{e^{-\omega_{o} \beta}\left\{\alpha \sin \left(\omega_{o} \alpha\right)-\beta \cos \left(\omega_{o} \alpha\right)\right\}+\beta}{1-e^{-\omega_{o} \beta}}=0.5,
\end{aligned}
$$

for $\alpha=2 r / c-t_{0.5}$. Rearranging we get

$e^{-\omega_{o} \beta}\left\{\alpha \sin \left(\omega_{o} \alpha\right)-\beta \cos \left(\omega_{o} \alpha\right)\right\}+\beta=0.5 \frac{\left[1-e^{-\omega_{o} \beta}\right]\left(\alpha^{2}+\beta^{2}\right)}{\beta}$.

This is a non-linear equation, where $\alpha$ can be determined by either a gradient optimization method or a graphical scheme. In a graphical scheme, the right-hand and left-hand sides are plotted as a function of $\alpha$, and the intersection points are identified. However, our goal is to generate rule-of-thumb formulae for the two limiting cases of large $Q$ and small $Q$.

\section{A1 Limiting case for small Q}

Assume $Q$ is small and $\omega_{o}$ is large so that $\omega_{o} \beta \gg 1$, where $\beta=r / c Q$. If $\alpha \omega_{0}=n \pi+\Delta \alpha$ for some large value $n \in[2,4,6, \ldots]$ and $\Delta \alpha$ is nearly zero, then eq. (A3) reduces to

$e^{-\omega_{o} \beta}\{\alpha \Delta \alpha-\beta\}+\beta=0.5 \frac{\left[1-e^{-\omega_{o} \beta}\right]\left(\alpha^{2}+\beta^{2}\right)}{\beta}+O\left(\Delta \alpha^{2}\right)$.

Substituting $\Delta \alpha=\alpha \omega_{0}-n \pi$ and rearranging we get to first-order in $\Delta \alpha$ :

$$
\begin{aligned}
0= & e^{-\omega_{o} \beta}\left\{\left(\alpha^{2} \omega_{0}-\alpha n \pi\right)-\beta\right\}+\beta-0.5 \frac{\left[1-e^{-\omega_{o} \beta}\right]\left(\alpha^{2}+\beta^{2}\right)}{\beta}, \\
= & \overbrace{\left[\omega_{o} e^{-\omega_{o} \beta}-\frac{\left\{1-e^{-\omega_{o} \beta}\right\}}{2 \beta}\right]}^{a} \alpha^{2}+\overbrace{\left[-n \pi e^{-\omega_{o} \beta}\right]}^{b} \alpha \\
& +\overbrace{0.5\left\{1-e^{-\omega_{o} \beta}\right\} \beta}^{c} .
\end{aligned}
$$

The solution to this quadratic equation is

$\alpha=\frac{n \pi e^{-\omega_{o} \beta} \pm \sqrt{n^{2} \pi^{2} e^{-2 \omega_{o} \beta}-4 a c}}{2\left[\omega_{o} e^{-\omega_{o} \beta}-\frac{\left\{1-e^{-\omega_{o} \beta}\right\}}{2 \beta}\right]}$, 
where

$4 a c=\left[\left(1+2 \omega_{o} \beta\right) e^{-\omega_{o} \beta}-1\right]\left[1-e^{-\omega_{o} \beta}\right]$.

Since $|\alpha|=\left|t_{0.5}-2 r / c\right|$ is the half width of the arrival then the full width, that is, the attenuation stretch $T^{\text {atten }}$, is just twice this value:

$T^{\mathrm{atten}}=\left|\frac{n \pi e^{-\omega_{o} \beta} \pm \sqrt{n^{2} \pi^{2} e^{-2 \omega_{o} \beta}-4 a c}}{\left[\omega_{o} e^{-\omega_{o} \beta}-\frac{\left\{1-e^{-\omega_{o} \beta}\right\}}{2 \beta}\right]}\right|$.

For large $\omega_{0} \beta \gg 0$ we have

$\lim _{\omega_{0} \beta \gg 0} T^{\text {atten }}=2 \beta=\frac{2 r}{c Q}$,

which is identical to that in eq. (13) for a highly lossy medium.

\section{A2 Limiting case for large $Q$}

For large $Q$, L'Hospital's says that the ratio in eq. (A2) reduces to

$$
\begin{aligned}
\lim _{Q \rightarrow \text { large }} \frac{d\left(\mathbf{g}, t_{0.5}\right)}{d\left(\mathbf{g}, t_{\text {peak }}\right)} & =\frac{\sin \left(\omega_{o} \alpha\right)}{\alpha \omega_{o}}, \\
& =0.5 .
\end{aligned}
$$

Thus, the half-width time interval $\alpha$ is $0.3017 T_{o}$ for a sinc function, so that the width of the sinc function is approximately $0.6 T_{o}$, which is identical to the lossless stretch factor $T^{\text {atten }}=0.6 T_{o}$ in eq. (14).

\section{APPENDIX B: EQUIVALENCY BETWEEN WAVEFORM GRADIENT AND MIGRATION}

The equivalency between the equations of the waveform gradient and migration can be shown by defining the data misfit function for waveforms to be

$\epsilon=\frac{1}{2} \int_{-\infty}^{\infty} \mathrm{d} \omega \sum_{i=1}^{N}\left(u_{i}-u_{i}^{\mathrm{obs}}\right)^{*}\left(u_{i}-u_{i}^{\mathrm{obs}}\right)$, where $u_{i}$ is the $i$ th predicted trace for a fixed frequency, the summation is over all $N$ traces located at geophones positions $\mathbf{g}$ on the surface, and $u_{i}^{\text {obs }}$ is the observed trace. Here we assume only reflected events in the traces and traces are integrated over all the useful frequencies in the data.

The misfit gradient with respect to the slowness $s(\mathbf{x})$ is given by

$\frac{\partial \epsilon}{\partial s(\mathbf{x})}=\int_{-\infty}^{\infty} \mathrm{d} \omega \sum_{i} \operatorname{Real}[\overbrace{\frac{\partial u_{i}}{\partial s(\mathbf{x})}}^{\text {Fréchet derivative }} \overbrace{\left(u_{i}-u_{i}^{\text {obs }}\right)^{*}}^{\text {data residual }}]$,

where the Fréchet derivative (Luo \& Schuster 1991; Li et al. 2017) for a source at the geophone position $\mathbf{g}_{i}$ is

$$
\begin{aligned}
\frac{\partial u_{i}}{\partial s(\mathbf{x})} & =-2 s(\mathbf{x}) \omega^{2} G\left(\mathbf{x} \mid \mathbf{g}_{i}\right)^{2}, \\
& \approx-2 s(\mathbf{x}) \omega^{2} A\left(\mathbf{g}_{i}, \mathbf{x}\right) e^{i \omega \tau_{g x g}} .
\end{aligned}
$$

where the trial image point is at $\mathbf{x}$, and $A\left(\mathbf{g}_{i}, \mathbf{x}\right)$ accounts for poststack geometric spreading in the far-field approximation. Denoting the second-time derivative of the scaled time-domain residual to be $d\left(\mathbf{g}_{i}, t\right)=-2 s(\mathbf{x}) A\left(\mathbf{g}_{i}, \mathbf{x}\right) \mathcal{F}^{-1}\left(\omega^{2}\left[u_{i}-u_{i}^{\text {obs }}\right]\right)$ and inserting eq. (B3) into eq. (B2) gives the post-stack gradient

$\frac{\partial \epsilon}{\partial s(\mathbf{x})}=\sum_{i} d\left(\mathbf{g}_{i}, \tau_{g x g}\right)$,

where $\mathcal{F}^{-1}$ [ ] represents the inverse Fourier transform operation. which is identical to the migration equation in eq. (1). Therefore, the gradient for linearized waveform inversion is equivalent to the migration of the residual traces. This means that the resolution formulae for migration are applicable to linearized waveform inversion images as long as non-zero-offset between the sources and receivers is accounted for in the resolution formulae.

Note, there is an implicit source wavelet deconvolution in waveform inversion which decreases the dominant period in the data. Therefore, the dominant source period in a linearized inversion image is equivalent to that after the source wavelet is deconvolved. 